\title{
Smooth Muscle Nitric 0xide Responsiveness and Clinical Maturation of Hemodialysis Arteriovenous Fistulae
}

\author{
Xiaoyong Tong, ${ }^{* \dagger}$ Xiuyun Hou, ${ }^{\dagger}$ Christopher Wason, ${ }^{\dagger}$ Tal Kopel, ${ }^{\ddagger}$ Richard A. Cohen, ${ }^{\dagger}$ and Laura M. Dember ${ }^{\S థ}$
}

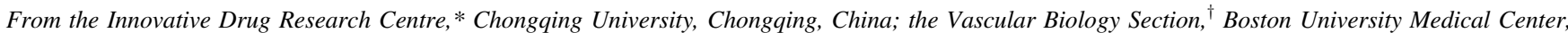
Boston, Massachusetts; the Nephrology Division, ${ }^{\ddagger}$ University of Montreal Hospital Centre, Hopital Saint-Luc, Montreal, Quebec, Canada; and the Renal, Electrolyte and Hypertension Division, ${ }^{\S}$ and the Center for Clinical Epidemiology and Biostatistics, ${ }^{\circledR}$ Perelman School of Medicine, University of Pennsylvania, Philadelphia, Pennsylvania
\end{abstract}

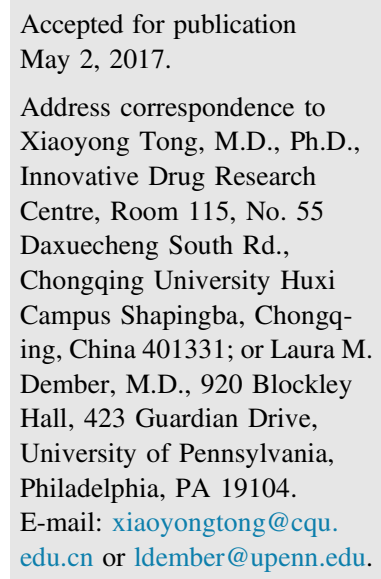

\begin{abstract}
The arteriovenous fistula is the preferred type of hemodialysis vascular access for patients with endstage renal disease, but a high proportion of newly created fistulas fail to mature for use. Stenosis caused by neointimal hyperplasia often is present in fistulas with maturation failure, suggesting that local mechanisms controlling vascular smooth muscle cell (SMC) migration and proliferation are important contributors to maturation failure. SMCs cultured from explants of vein tissue obtained at the time of fistula creation from 19 patients with end-stage renal disease were studied to determine whether smooth muscle responsiveness to nitric oxide is associated with fistula maturation outcomes. Nitric oxide-induced inhibition of smooth muscle cell migration, but not proliferation, was greater in cells from patients with subsequent fistula maturation success than from patients with subsequent fistula maturation failure (mean inhibition percentage, 17 versus 5.7, respectively; $P=0.035$ ). Impaired nitric oxide responsiveness was associated with oxidation of the calcium regulatory protein, sarcoplasmic/endoplasmic reticulum calcium ATPase (SERCA), and was reversed by overexpressing SERCA (1.8-fold increase in inhibition, $P=0.0128$ ) or down-regulating Nox4-based NADPH oxidase (2.3-fold increase in inhibition; $P=0.005$ ). Our data suggest that the nitric oxide responsiveness of SMC migration is associated with fistula maturation success and raises the possibility that therapeutic restoration of nitric oxide responsiveness through manipulation of local mediators may prevent fistula maturation failure. (Am J Pathol 2017, 187: 2095-2101; http://dx.doi.org/10.1016/ j.ajpath.2017.05.006)
\end{abstract}

Approximately 500,000 Americans have end-stage renal disease and most of these patients are treated with hemodialysis. Inadequate vascular access for hemodialysis is a major cause of morbidity and mortality, with associated costs in the United States exceeding 1.5 billion dollars per year. $^{1}$ An arteriovenous fistula is the preferred type of vascular access because of its lower complication rates compared with synthetic arteriovenous grafts or central venous catheters. However, up to $60 \%$ of newly created fistulas never become usable for dialysis because they fail to mature, meaning that the vessels do not undergo the enlargement necessary to support the dialysis blood circuit. $^{2,3}$ There currently are no interventions known to prevent fistula maturation failure. Excessive neointimal hyperplasia increasingly is recognized as an important cause of maturation failure. ${ }^{4,5}$ Thus, it is likely that local inflammatory and oxidant mechanisms that accelerate the migration and proliferation of the vascular smooth muscle cells (SMCs) that constitute the neointima are key mediators

Supported by National Natural Science Foundation of China grant 31571172 (X.T.), Chongqing Research Program of Basic Research and Frontier Technology grant cstc2016jcyjA0407 (X.T.), American Diabetes Association award 7-09-JF-69 (X.T.) and NIH grants R21DK084390 (R.A.C., X.T., and L.M.D.), R01 HL031607 (R.A.C. and X.T.), R37 HL104017 (R.A.C.), and U01 DK082232 (L.M.D.).

Disclosures: None declared. 
of fistula maturation failure. ${ }^{6-8} \mathrm{We}$ hypothesized that local factors that impair SMC responsiveness to the endothelium-derived vasodilator, nitric oxide, are important contributors to maturation failure. By using SMCs cultured from the veins used to create hemodialysis fistulae in patients with end-stage renal disease, we found that fistula maturation failure was associated with impaired responsiveness of SMC migration to nitric oxide, a property that we have shown in animal models to be the result of oxidation of the key calcium regulatory protein, the sarcoplasmic/endoplasmic reticulum calcium ATPase (SERCA). Furthermore, we showed that abnormal nitric oxide responsiveness can be reversed by overexpression of SERCA or down-regulation of Nox4-based NADPH oxidase (Nox4), a major reactive oxygen species-generating enzyme known to cause oxidation of SERCA. ${ }^{9,10}$

\section{Materials and Methods}

\section{Participants and Clinical Outcomes}

This was an ancillary study of the National Institute of Diabetes and Digestive and Kidney Diseases Hemodialysis Fistula Maturation Study, a multicenter observational study that was designed to elucidate predictors and underlying mechanisms of hemodialysis fistula maturation, ${ }^{11}$ and included both a subset of the Hemodialysis Fistula Maturation Study participants as well as patients who underwent fistula creation surgery before the initiation of the Hemodialysis Fistula Maturation Study. Patients undergoing creation of an arteriovenous fistula at Boston University were enrolled. At the time of fistula creation, a circumferential segment of vein that was approximately $5 \mathrm{~mm}$ in length was obtained and processed for immunohistochemistry, SMC culture, and gene expression analysis. The fistula clinical maturation outcome was ascertained using the criteria developed by the National Institute of Diabetes and Digestive and Kidney Diseases Dialysis Access Consortium, which required successful use of the fistula for $75 \%$ of hemodialysis sessions during the fifth month after fistula creation. ${ }^{3,12}$ The individuals who ascertained the clinical maturation outcomes did not have access to the in vitro or tissue data, and, conversely, the individuals performing the laboratory experiments were not aware of the clinical maturation outcomes. This study was approved by the Boston University Institutional Review board (protocols $\mathrm{H}-26674$ and $\mathrm{H}-28837$ ), and all participants provided written informed consent.

\section{Human Venous SMC Culture}

The explant method was used to culture SMCs from fragments of vein obtained during fistula creation surgery. The media of the vessel was cut into $1-\mathrm{mm}^{2}$ pieces and placed onto collagen I-coated plastic tissue culture plates in Dulbecco's modified Eagle's medium supplemented with

Table 1 Baseline Characteristics

\begin{tabular}{|c|c|c|c|}
\hline Characteristic & All $(n=19)$ & $\begin{array}{l}\text { Fistula maturation } \\
\text { success }(n=9)\end{array}$ & $\begin{array}{l}\text { Fistula maturation } \\
\text { failure }(n=10)\end{array}$ \\
\hline Age, years & $56.2 \pm 10.7$ & 55.9 & 56.9 \\
\hline \multicolumn{4}{|l|}{ Race } \\
\hline Black & $14(73.7)$ & $5(55.6)$ & $9(90.0)$ \\
\hline White & $3(15.8)$ & $2(22.2)$ & $1(10.0)$ \\
\hline Hypertension & $17(89.5)$ & $8(88.9)$ & $9(90.0)$ \\
\hline Diabetes & $8(42.1)$ & $2(22.2)$ & $6(60.0)$ \\
\hline Hyperlipidemia & $10(52.6)$ & $6(66.7)$ & $4(40.0)$ \\
\hline Vascular disease* & $9(47.4)$ & $3(33.3)$ & $6(60.0)$ \\
\hline \multicolumn{4}{|l|}{ Medications } \\
\hline Upper arm & $12(63.2)$ & $6(66.7)$ & $6(60.0)$ \\
\hline \multicolumn{4}{|l|}{ Vein morphometry ${ }^{\dagger}$} \\
\hline Whole vein, $\mathrm{mm}^{2}$ & $5.74 \pm 2.88$ & $4.94 \pm 2.33$ & $6.14 \pm 3.18$ \\
\hline Lumen, $\mathrm{mm}^{2}$ & $1.79 \pm 1.22$ & $2.01 \pm 0.75$ & $1.68 \pm 1.44$ \\
\hline
\end{tabular}

Data are expressed as means \pm SD or $n(\%) . P>0.05$ for all comparisons. Maturation success and failure groups were compared with $t$-tests or the Fisher exact test.

*Defined as a history of any of the following: myocardial infarction, angina, coronary artery bypass surgery or angioplasty, congestive heart failure, cardiac arrhythmias or conduction problems, stroke, transient ischemic attack, carotid endarterectomy, carotid artery angioplasty, lower-extremity arterial bypass surgery or angioplasty, nontraumatic amputation, and claudication.

${ }^{\dagger}$ Cross-sectional areas were measured from vein tissue obtained at the time of fistula creation. 
$20 \mathrm{mmol} / \mathrm{L}$ L-glutamine and HEPES buffer, $100 \mathrm{U} / \mathrm{mL}$ penicillin, and $100 \mu \mathrm{g} / \mathrm{mL}$ streptomycin, containing $10 \%$ fetal calf serum in a humidified atmosphere $\left(37^{\circ} \mathrm{C} ; 95 \%\right.$ air-5\% $\mathrm{CO}_{2}$ ). SMC phenotypes were confirmed using a fluorescein isothiocyanate-tagged anti- $\alpha$-smooth muscle actin antibody. SMCs were used up to passage five based on our preliminary studies that showed a similar migratory rate with passages two through five.

\section{Determination of PDGF-Induced Cell Proliferation and the Response to Nitric Oxide}

A tetrazolium-based nonradioactive proliferation assay kit (Quick Cell Proliferation Assay Kit II; BioVision, Milpitas, CA) was used to determine the cell number according to the manufacturer's protocol. ${ }^{13}$ Cells were seeded on 96-well plates at a density of $5 \times 10^{3}$ cells per well in media containing $5 \mathrm{ng} / \mathrm{mL}$ platelet-derived growth factor (PDGF) with or without $300 \mu \mathrm{mol} / \mathrm{L}$ nitric oxide donor, diethylenetriamine (DETA) NONOate (Cayman Chemical, Ann Arbor, $\mathrm{MI}$ ), and $0.2 \%$ fetal bovine serum medium was used as a control. Culture medium was changed daily. The cell number in each well was determined 72 hours later.

\section{Determination of PDGF-Induced Migration and the Response to Nitric Oxide}

Migration was determined with a simple cell monolayer wounding assay ${ }^{9,10,14}$ with media containing $5 \mathrm{ng} / \mathrm{mL}$ PDGF with or without $300 \mu \mathrm{mol} / \mathrm{L}$ nitric oxide donor DETA NONOate. Photographs were taken at 0 and 6 hours at three fixed locations along the scratch with a light microscope and analyzed using ImageJ software version 1.51 (NIH, Bethesda, MD; http://imagej.nih.gov/ij).

\section{Immunohistochemistry}

Immunohistochemistry was performed on small fragments of vein obtained at the time of fistula creation after fixation in $10 \%$ buffered formalin, dehydration, and paraffinembedding. ${ }^{15,16}$ Sections were stained with SERCA cysteine-674- $\mathrm{SO}_{3} \mathrm{H}$ antibody or Nox4 antibody individually and developed with the Vectastain $\mathrm{ABC}$ kit (Vector, Burlingame, CA) followed by counterstaining with hematoxylin. Negative controls were obtained with the appropriate IgG isotype. Three independent observers scored the staining intensity from images using a scale of 0 to $4 .^{10}$ Morphologic analysis was performed only on fully circumferential vein samples using a modified Movat's stain. The areas of outer vein and its lumen were determined using ImageJ. The mean cross-sectional areas of neointimal and medial areas were determined. The medial area was calculated as the area encircled by the external elastic lamina minus the area encircled by the internal elastic lamina. The intima to media ratio was quantified by dividing the neointimal area by the medial area.
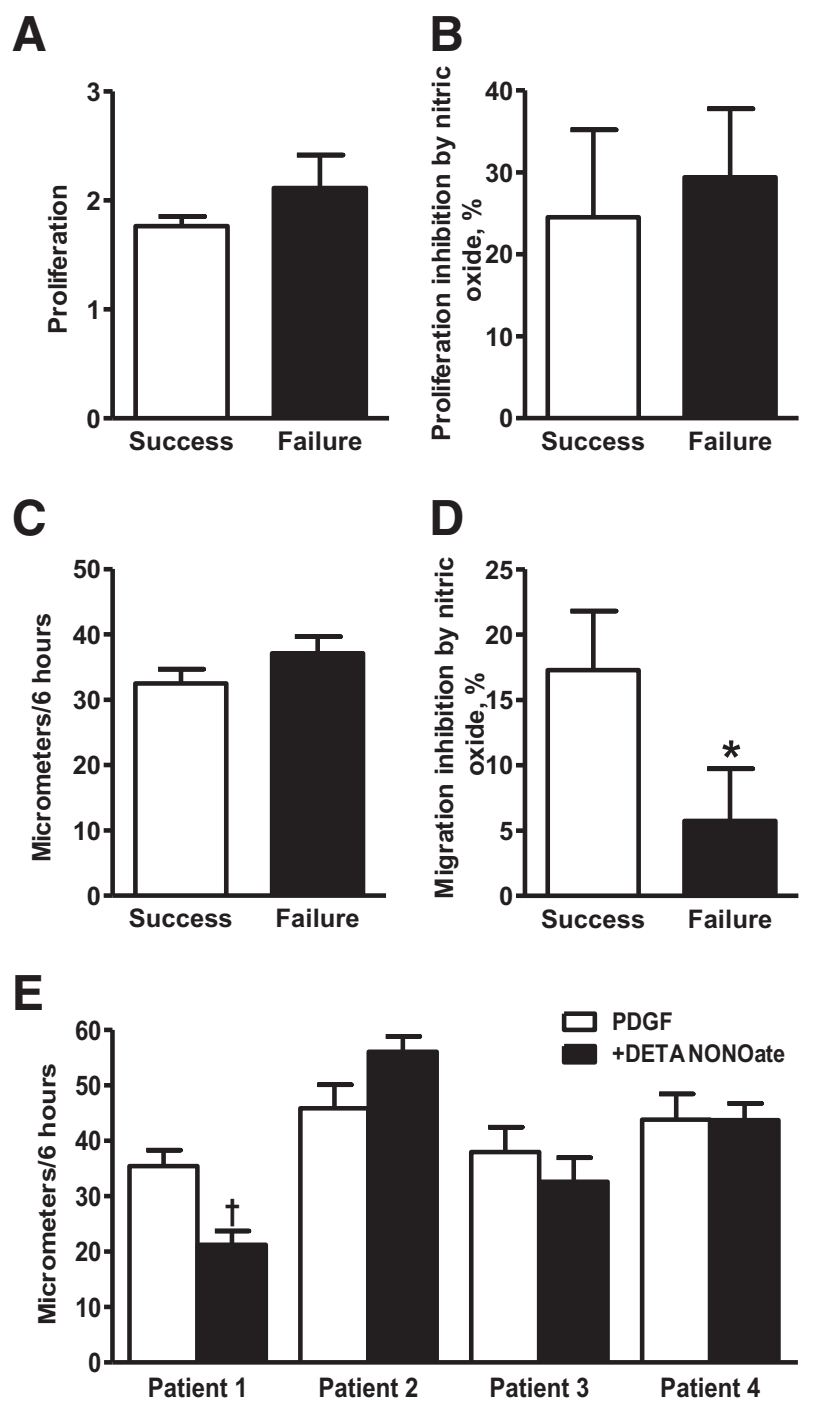

Figure 1 Fistula clinical maturation outcome is associated with nitric oxide response in culture. A: Proliferation in response to platelet-derived growth factor (PDGF) of smooth muscle cells (SMCs) from patients with subsequent fistula maturation success or failure. Cell proliferation is displayed as ratio to control. B: Responsiveness to nitric oxide of SMC proliferation for the same samples as in A. C and $\mathbf{D}$ : Migration in response to PDGF of SMCs from patients with subsequent fistula maturation success or failure (C). Inhibition of migration by diethylenetriamine (DETA) NONOate for SMCs from the same patients (D). E: Venous SMC migration in response to PDGF alone or PDGF plus DETA NONOate are shown for the first four patients studied (patients 1 to 4 ). $n=4$ to $8(\mathbf{A}) ; n=9$ to 10 (C and $\mathbf{D}$ ); $n=5$ to 6 (E, analyses per patient cells). ${ }^{\star} P<0.05$ versus the maturation success group ( $U$-test) $;{ }^{\dagger} P<0.05$ versus PDGF (Bonferroni post-tests after two-way analysis of variance).

\section{Real-Time Quantitative PCR}

Total cellular RNA was isolated from SMCs cultured in $0.2 \%$ fetal bovine serum and Dulbecco's modified Eagle's medium for 24 hours and retrotranscribed to cDNA. Realtime quantitative PCR was performed with synthetic TaqMan gene-specific primers (Applied Biosystems, Foster city, CA) according to the following cycling conditions: denaturation, annealing, and extension at $95^{\circ} \mathrm{C}, 55^{\circ} \mathrm{C}$, and 
$72^{\circ} \mathrm{C}$ for 30 seconds, 30 seconds, and 1 minute, respectively, for 40 cycles. $18 \mathrm{~S}$ ribosomal RNA was used as an internal control.

\section{Adenoviral Transfection of SMCs}

For some migration assays, SMCs were infected with adenovirus to overexpress SERCA $2 b$ or with an adenovirus with an empty vector, Nox4 siRNA, ${ }^{17}$ or its control siRNA at a multiplicity of infection of 50 in $0.2 \%$ fetal calf serum medium for 3 days before the migration assay.

\section{Statistical Analysis}

All data are presented as means \pm SEM unless otherwise specified. A $P$ value of $<0.05$ was considered statistically significant.

\section{Results}

\section{Baseline Characteristics of Patients}

Baseline characteristics of the patients, vein and lumen areas, and fistula configurations are shown in Table 1, stratified by fistula maturation outcome.

\section{Fistula Clinical Maturation Outcome Is Associated with Nitric 0xide Responsiveness of SMC Migration in Culture}

Because intimal hyperplasia is an important feature of a failed arteriovenous fistula, we measured SMC proliferation and migration. Cultured SMCs from patients with subsequent fistula maturation failure and SMCs from patients with subsequent maturation success behaved similarly regarding PDGF-induced proliferation, and proliferation was inhibited similarly by the NO donor DETA NONOate (Figure 1, A and B). SMC migration also was not significantly different for cells from the two groups of patients (Figure 1C), however, the inhibitory effect of nitric oxide on SMC migration was decreased significantly in cells from patients with subsequent maturation failure (Figure 1D). This result illustrates a range of responses to nitric oxide by venous SMCs from patients with end-stage renal disease. Figure 1E shows the SMC nitric oxide response for the first four patients studied. Despite migration responses to PDGF that were similar among the four patients, DETA NONOate inhibited SMC migration from patient 1 (subsequent maturation success), but not from patients 2 to 4 (subsequent maturation failures).

Increased Inflammation and 0xidation in Veins from Patients with Subsequent Fistula Maturation Failure

Based on our previous studies indicating that cysteine-674 of SERCA is the major cysteine regulated by nitric oxide, and that its irreversible oxidation $\left(\mathrm{C} 674-\mathrm{SO}_{3} \mathrm{H}\right)$ leads to impaired inhibition of SMC migration by nitric oxide,, ,10,14 we examined the relationship between nitric oxide responsiveness and SERCA cysteine-674 oxidation in human vein samples. Figure 2A shows immunohistochemistry performed on the vein samples from the first four patients studied (Figure 1D). In contrast to the vein from patient 1 (maturation success), the veins from patients 2 to 4 (maturation failures) had intense SERCA $\mathrm{C} 674-\mathrm{SO}_{3} \mathrm{H}$, stained red, indicating greater irreversible sulfonic acid oxidation of
A

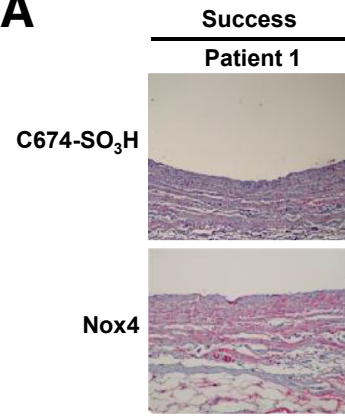

C

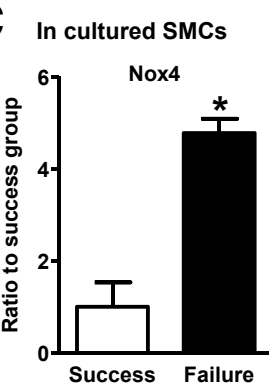

Failure

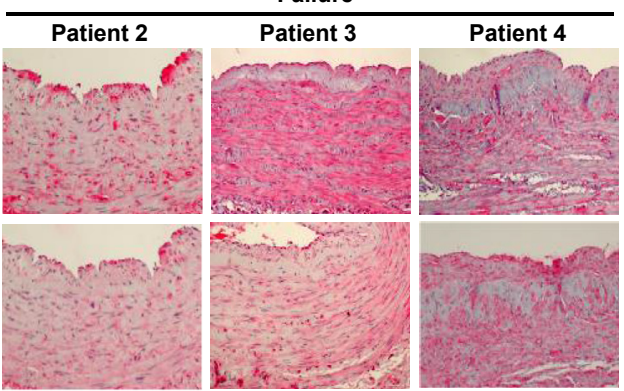

B

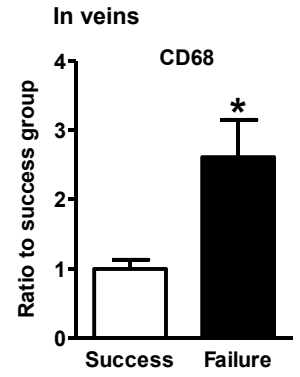

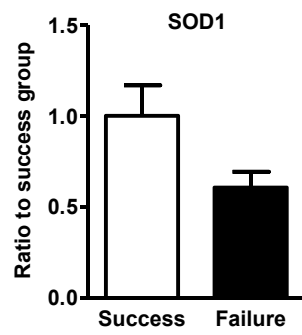

Figure 2 Increased inflammation and oxidation in failed maturation veins. A: Immunohistochemistry of vein tissue obtained at fistula creation of the first four patients studied using antibody against sarcoplasmic/endoplasmic reticulum calcium ATPase (SERCA) cysteine-674- $\mathrm{SO}_{3} \mathrm{H}\left(\mathrm{C}_{674}-\mathrm{SO}_{3} \mathrm{H}\right)$ or Nox4. Red indicates reactivity with the antibody. The counterstain with hematoxylin appears blue. B: CD68 and superoxide dismutase 1 (SOD1) mRNA levels in veins. C: Nox4 and Toll-like receptor 4 (TLR4) mRNA levels in cultured smooth muscle cells (SMCs). $n=5$ CD68 patient samples (B); $n=3$ to 6 SOD1 patient samples (B); $n=3$ to 5 patient samples (C). $P=0.09$ for S0D1 mRNA expression in maturation failure versus success (B). ${ }^{*} P<0.05$ versus maturation success group (unpaired $t$-test). Original magnification, $\times 4($ A). 
A

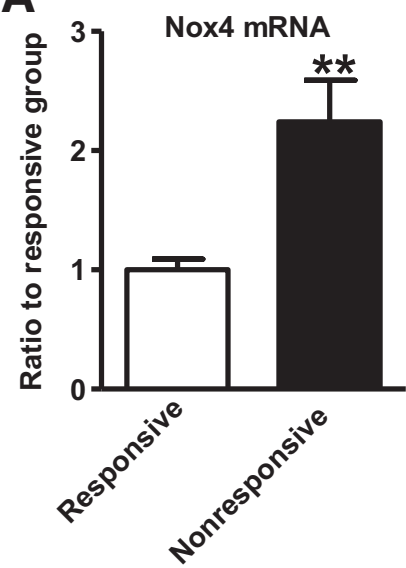

B

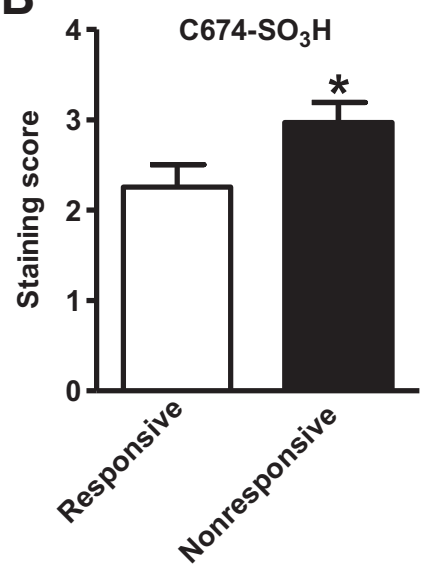

C

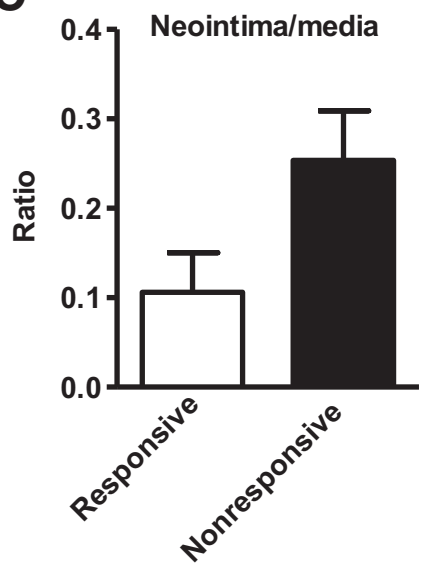

Figure 3 Increased Nox4 and sarcoplasmic/endoplasmic reticulum calcium ATPase (SERCA) irreversible oxidation in patients with smooth muscle cell (SMC) nitric oxide nonresponsiveness. A: Nox4 mRNA levels in vein tissue from patients with nitric oxide-responsive SMCs, defined as migration inhibition by diethylenetriamine NONOate greater than or equal to the median value of $14.19 \%$, or nitric oxide-nonresponsive SMCs (<14.19\%). B: SERCA C674-S0 ${ }_{3} \mathrm{H}$ staining scores (scale, 0 to 4 ) in vein tissue from patients with nitric oxide-responsive SMCs or nitric oxide-nonresponsive SMCs, again defined as greater than or equal to the median value or less than the median value, respectively. C: Neointimal area to media area ratio in vein tissue from patients with nitric oxide-responsive SMCs or nitric oxide-nonresponsive SMCS, again defined as greater than or equal to the median value or less than the median value, respectively. $n=5$ to 7 patient samples $(\mathbf{A}) ; n=9$ to 10 patient samples $(\mathbf{B}) ; n=6$ to 8 patient samples (C). $P=0.061$ nonresponsive versus responsive (U-test) $(\mathbf{A}) .{ }^{*} P<0.05,{ }^{*} P<0.01$ versus responsive (unpaired $t$-test).

SERCA cysteine-674. ${ }^{10,15}$ Nox4 is the major reactive oxygen species-generating enzyme in the vasculature. We previously found in several animal models of disease that increased Nox 4 is responsible for the irreversible oxidation of SMC SERCA cysteine-674 and the associated impairment in nitric oxide response. 9,10 The pattern of Nox4 staining in the human vein samples was similar to that of SERCA C674- $\mathrm{SO}_{3} \mathrm{H}$ (Figure 2A). The staining of both oxidized SERCA and Nox4 was present throughout the vein wall, which included both neointimal cells in patients 2 to 4 and the native smooth muscle layers. Next, we measured inflammation makers and antioxidant enzyme levels in veins because they indicate the oxidant state of the vein. The macrophage marker CD68 was increased significantly and the antioxidant enzyme superoxide dismutase 1 decreased in the veins from patients with subsequent maturation success compared with patients with subsequent maturation failure (Figure 2B). In cultured SMCs, both Nox4 and the inflammation marker Toll-like receptor 4 were increased significantly in cells from patients with subsequent maturation success compared with patients with subsequent maturation failure (Figure 2C).

\section{Increased Nox4 and SERCA Irreversible 0xidation in Patients with SMC Nitric Oxide Nonresponsiveness}

Figure 3, A and B show SERCA C674- $\mathrm{SO}_{3} \mathrm{H}$ staining scores and Nox 4 mRNA expression levels in the vein tissue from patients who later were determined to have SMC nitric oxide responsiveness and nonresponsiveness, defined by the median value of migration inhibition by DETA NONOate in culture. The mRNA level of Nox4 and the staining score of
SERCA C674- $\mathrm{SO}_{3} \mathrm{H}$ were increased in the tissue from patients with SMC nitric oxide nonresponsiveness compared with patients with nitric oxide responsiveness, suggesting that the poor nitric oxide response might be because of the up-regulation of Nox4 and increased irreversible oxidation of SERCA cysteine-674 (Figure 3, A and B). Although not reaching statistical significance, the ratio of the neointima area to the media area in veins from patients with SMC nitric oxide nonresponsiveness was twice that in veins yielding SMCs with nitric oxide responsiveness (Figure 3C).
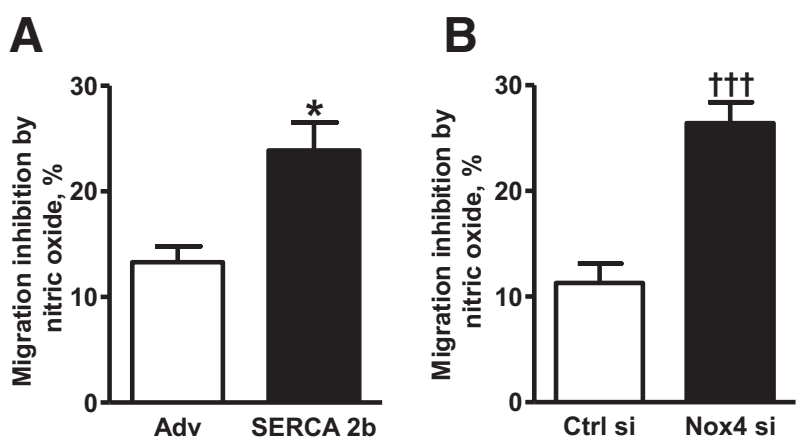

Figure 4 Smooth muscle cell (SMC) nitric oxide responsiveness after overexpression of sarcoplasmic/endoplasmic reticulum calcium ATPase (SERCA) $2 b$ or down-regulation of Nox4. A: Overexpression of SERCA $2 b$ improves nitric oxide response in cultured venous SMCs from the patients with nitric oxide-nonresponsive SMCs, defined as less than $14.19 \%$ migration inhibition by diethylenetriamine (DETA) NONOate. B: Downregulation of Nox4 improves nitric oxide response in cultured venous SMCs from the patients with nitric oxide-nonresponsive SMCs, defined as less than $14.19 \%$ migration inhibition by DETA NONOate. $n=10$ patient samples. ${ }^{*} P<0.05$ versus adenovirus control (unpaired $t$-test); ${ }^{\dagger \dagger} P<0.001$ versus adenovirus control siRNA (unpaired $t$-test). Adv, adenovirus; Ctrl si, control siRNA. 
Overexpression of SERCA $2 \mathrm{~b}$ or Down-Regulation of Nox4 Restores Nitric 0xide Responsiveness in Cultured Venous SMCs

To evaluate the impact of SERCA cysteine-674 oxidation and Nox4 up-regulation on SMC nitric oxide responsiveness further, we overexpressed wild-type SERCA $2 b$ and downregulated Nox4 as strategies for improving nitric oxide responsiveness. SMC nitric oxide responsiveness was restored by both of these manipulations (Figure 4).

\section{Discussion}

Our findings suggest the possibility that increased Nox4 expression and SERCA oxidation in the uremic milieu of end-stage renal disease impair nitric oxide function and may contribute to intimal hyperplasia before fistula creation, and that these characteristics can also influence nitric oxide responsiveness of migrating SMCs in culture. In culture, differences were not observed in migratory or proliferative responses to PDGF or in the inhibition of proliferation by nitric oxide. However, our results point to poor nitric oxide responsiveness of migrating SMCs derived from veins as a property that is sustained in culture and that also may contribute to intimal hyperplasia and fistula maturation failure in vivo after fistula creation. In patients with SMCs that had poor nitric oxide responsiveness, overexpression of wild-type SERCA 2b or down-regulation of Nox4 improved nitric oxide responsiveness. Based on our previous work, ${ }^{9,10,14}$ this finding in human cells may indicate that despite substantial oxidation of endogenous SERCA in diseased cells, sufficient nonoxidized SERCA can be expressed to respond to nitric oxide, and thereby decrease $\mathrm{Ca}^{2+}$ and inhibit SMC migration. ${ }^{10,14}$ We recently showed in rodents that SMC Nox4 is involved directly in the development of neointimal hyperplasia and atherosclerosis, and that down-regulation of SMC Nox4 improves vessel function under pathologic conditions. ${ }^{13,18}$ The finding that Nox4 knockdown restores nitric oxide responsiveness of SMCs harvested from patients with end-stage renal disease suggests that the processes may be similar in humans. Interestingly, our results are consistent with the finding from the full Hemodialysis Fistula Maturation cohort that preoperative nitroglycerin-mediated brachial artery dilation, an in vivo indicator of nitric oxide responsiveness, is associated with fistula vein diameter and blood flow after surgery, both of which are key components of fistula clinical maturation. ${ }^{19}$

In summary, our data suggest that SMC nitric oxide responsiveness is associated with successful fistula maturation and that restoration of nitric oxide responsiveness through manipulation of local factors may be a promising therapeutic strategy. The superficial location and accessibility of the fistula make it highly suited for application of local drug or gene therapies, both during and after its surgical creation. The findings from these studies also may have applicability to the more general problem of impaired nitric oxide responsiveness associated with kidney failure and cardiovascular disease.

\section{References}

1. NIH: U.S. Renal Data System. USRDS 2011 Annual Data Report: Atlas of Chonic Kidney Disease and End-Stage Renal Disease in the United States. Bethesda, MD: National Institutes of Health, National Institute of Diabetes and Digestive and Kidney Diseases, 2011

2. Allon M, Robbin ML: Increasing arteriovenous fistulas in hemodial ysis patients: problems and solutions. Kidney Int 2002, 62:1109-1124

3. Dember LM, Beck GJ, Allon M, Delmez JA, Dixon BS, Greenberg A, Himmelfarb J, Vazquez MA, Gassman JJ, Greene T, Radeva MK, Braden GL, Ikizler TA, Rocco MV, Davidson IJ, Kaufman JS, Meyers CM, Kusek JW, Feldman HI: Effect of clopidogrel on early failure of arteriovenous fistulas for hemodialysis: a randomized controlled trial. JAMA 2008, 299:2164-2171

4. Wali MA, Eid RA, Dewan M, Al-Homrany MA: Pre-existing histopathological changes in the cephalic vein of renal failure patients before arterio-venous fistula (AVF) construction. Ann Thorac Cardiovasc Surg 2006, 12:341-348

5. Roy-Chaudhury P, Arend L, Zhang J, Krishnamoorthy M, Wang Y, Banerjee R, Samaha A, Munda R: Neointimal hyperplasia in early arteriovenous fistula failure. Am J Kidney Dis 2007, 50:782-790

6. Roy-Chaudhury P, Sukhatme VP, Cheung AK: Hemodialysis vascular access dysfunction: a cellular and molecular viewpoint. J Am Soc Nephrol 2006, 17:1112-1127

7. Dixon BS: Why don't fistulas mature? Kidney Int 2006, 70: $1413-1422$

8. Roy-Chaudhury P, Wang Y, Krishnamoorthy M, Zhang J, Banerjee R, Munda R, Heffelfinger S, Arend L: Cellular phenotypes in human stenotic lesions from haemodialysis vascular access. Nephrol Dial Transplant 2009, 24:2786-2791

9. Tong X, Schröder K: NADPH oxidases are responsible for the failure of nitric oxide to inhibit migration of smooth muscle cells exposed to high glucose. Free Radic Biol Med 2009, 47:1578-1583

10. Tong X, Hou X, Jourd'heuil D, Weisbrod RM, Cohen RA: Upregulation of Nox4 by TGF[beta]1 oxidizes SERCA and inhibits NO in arterial smooth muscle of the prediabetic Zucker rat. Circ Res 2010, 107:975-983

11. Dember LM, Imrey PB, Beck GJ, Cheung AK, Himmelfarb J, Huber TS, Kusek JW, Vazquez MA, Alpers CE, Robbin ML, Vita JA, Greene T, Gassman JJ, Feldman HI; Hemodialysis Fistula Maturation Study Group: Objectives and design of the hemodialysis fistula maturation study. Am J Kidney Dis 2014, 63:104-112

12. Dember LM, Kaufman JS, Beckb G, Dixon BS, Gassmanb J, Greeneb T, Himmelfarbd J, Hunsickerci LG, Kuseke JW, Lawsonf JH, Middletonf JP, Radevab M, Schwab SI, Whiting JF, Feldman HI; DAC Study Group: Design of the Dialysis Access Consortium (DAC) clopidogrel prevention of early AV fistula thrombosis trial. Clin Trials 2005, 2:413-422

13. Tong X, Khandelwal AR, Qin Z, Wu X, Chen L, Ago T, Sadoshima J, Cohen RA: Role of smooth muscle Nox4-based NADPH oxidase in neointimal hyperplasia. J Mol Cell Cardiol 2015, 89:185-194

14. Qin Z, Hou X, Weisbrod RM, Seta F, Cohen RA, Tong X: Nox2 mediates high fat high sucrose diet-induced nitric oxide dysfunction and inflammation in aortic smooth muscle cells. J Mol Cell Cardiol 2014, 72:56-63

15. Ying J, Sharov V, Xu S, Jiang B, Gerrity R, Schöneich C, Cohen RA: Cysteine-674 oxidation and degradation of sarcoplasmic reticulum $\mathrm{Ca} 2+$ ATPase in diabetic pig aorta. Free Radic Biol Med 2008, 45:756-762

16. Xu S, Ying J, Jiang B, Guo W, Adachi T, Sharov V, Lazar H, Menzoian J, Knyushko TV, Bigelow D, Schöneich C, Cohen RA: 
Detection of sequence-specific tyrosine nitration of manganese SOD and SERCA in cardiovascular disease and aging. Am J Physiol Heart Circ Physiol 2006, 290:H2220-H2227

17. Chen K, Kirber MT, Xiao H, Yang Y, Keaney JF: Regulation of ROS signal transduction by NADPH oxidase 4 localization. J Cell Biol 2008, 181:1129-1139

18. Tong X, Khandelwal AR, Wu X, Xu Z, Yu W, Chen C, Zhao W, Yang J, Qin Z, Weisbrod RM, Seta F, Ago T, Lee KSS, Hammock BD,
Sadoshima J, Cohen RA, Zeng C: Pro-atherogenic role of smooth muscle Nox4-based NADPH oxidase. J Mol Cell Cardiol 2016, 92: $30-40$

19. Allon M, Greene T, Dember LM, Vita JA, Cheung AK, Hamburg NM, Imrey PB, Kaufman JS, Robbin ML, Shiu Y, Terry CM, Umphrey HR, Feldman HI: Association between preoperative vascular function and postoperative arteriovenous fistula development. J Am Soc Nephrol 2016, 27:3788-3795 